\title{
Editorial
}

\section{Disparate Chronic Kidney Disease-Associated Outcomes in South Asians Ethnicity or Estimated Glomerular Filtration Rate?}

\author{
Gopesh K. Modi ${ }^{\mathrm{a}}$ Vivekanand Jha ${ }^{\mathrm{b}-\mathrm{d}}$ \\ a Samarpan Kidney Center, Bhopal, India; ${ }^{b}$ George Institute for Global Health, UNSW, New Delhi, India; \\ 'George Institute for Global Health, University of Oxford, Oxford, UK; d Manipal Academy of Higher Education, \\ Manipal, India
}

Chronic kidney disease (CKD) is currently ranked at the 16th place in the list of global years of lives lost by the Global Burden of Disease Study; it is projected to rise to rank 5 by the year 2040. This increase is faster in some parts of the world compared to others [1]. Contrary to the usual perception of its inevitable progression to endstage kidney failure, a substantial proportion of patients with CKD die of cardiovascular (CV) complications in earlier stages of disease. A remarkable body of work led by the CKD Prognosis Consortium and the CKD Epidemiology Collaboration, and using data from cohorts from around the world - has confirmed the relationship between common markers of kidney disease and the development of CV disease (CVD) and all-cause as well as CV mortality [2]. Both estimated glomerular filtration rate (eGFR) and albuminuria have independent as well as multiplicative impact on outcomes. These findings have been shown to be consistent in several populations around the world. Interestingly, there is no data so far on this association in South Asians, especially from India.

The Baseline Southall and Brent studies were set up in 1988-1991 to study the balance between genetic and environmental influences among migrants to the United Kingdom of South Asian (mostly Indian) and Afro-Ca- ribbean origins and the native UK population of European origin.

In this issue of the Journal, Eastwood et al. [4] report their findings on the association between kidney function markers (eGFR and urinary albumin-creatinine ratio $[\mathrm{ACR}])$ at baseline and incident CVD and mortality after 20 years and examine the ethnicity-specific differences between Indo-Asians and Europeans from Southall in this cohort [3]. About $30-40 \%$ of invited participants had declined to be enrolled in the original baseline study. The age at the time of recruitment was 40-69 years. Of the 3,754 participants from Southall, the current analysis included 2,220 participants (Indo-Asians: 1,104, Europeans: 1,116). eGFR, based both on creatinine (eGFRcreat) and cystatin (eGFRcys) measurements, and ACR were measured only at baseline. Outcome data were sourced from NHS Digital that allowed the ascertainment of deaths and CVD episodes, showing the value of data linkage [4].

The 2 ethnicities exhibited important baseline differences related to CV risk: South Asians had more adverse CV risk profile, including lower eGFRcys and ACR values. Of note, when participants placed under a certain CKD stage on the basis of eGFRcreat were reclassified based on eGFRcys values, South Asians were more likely to be designated to a more adverse CKD stage. The au-

\section{KARGER}

(C) 2019 S. Karger AG, Basel

E-Mail karger@karger.com

www.karger.com/ajn
Prof. Vivekanand Jha

George Institute for Global Health

310-11 Elegance Tower, Jasola District Centre

New Delhi 110025 (India)

E-Mail vjha@georgeinstitute.org.in 
thors found both eGFRcys and eGFRcreat to be associated with mortality and incident CVD in Europeans. However, this association was absent for eGFRcreat and eGFRcys in South Asians. In contrast, ACR was more strongly associated with outcomes in South Asians than it was among the Europeans. This finding was confirmed using several modelling approaches.

The study supports the narrative on the ethnic differences in biological behavior of diseases. Prima facie, it would seem that pathophysiology of CKD and its complications may be different in the 2 ethnic groups and may suggest the need to adopt a differential approach to assessment and management of CKD depending on ethnicity. A faster progression of CKD and higher albuminuria has been reported in South Asians and other ethnic groups in comparison to Caucasians [5].

However, a deeper look into the results and application of principles of biological plausibility is warranted. The authors have postulated eGFR and albuminuria as complimentary markers of kidney function, with albuminuria indicating the particular susceptibility of South Asians to microvascular disease and endothelial dysfunction. A differential impact of eGFR and albuminuria on CVD and mortality has been shown in the CKDPrognosis Consortium analyses [2]. eGFR shows a threshold effect, with the risk rising after eGFR dips below $70 \mathrm{~mL} / \mathrm{min} / 1.73 \mathrm{~m}^{2}$, whereas albuminuria has a continuous gradient of increasing risk starting from very low levels, even below the current definitions of microalbuminuria. Studies have shown that differences in the albumin excretion rates between individuals become evident at a very early age after birth and persist for decades suggesting a predefined state of endothelial/renal health [6]. This "level of endowment" of albuminuria also may indicate the variations in propensity to development of conditions like diabetes, hypertension, and CV disease later in life.

Since the eGFR did not predict outcomes as expected in South Asians, we should examine the issue of ethnicity-specific differences in GFR estimation. The equations the authors used for South Asians have been derived primarily in Caucasians, and are shown to be imprecise for many populations. The $2002 \mathrm{KDOQI}$ guidelines lay down a performance measure for accuracy requiring that the eGFR values derived from such an equation should be within $30 \%$ of the measured GFR obtained using a gold standard method in $>90 \%$ of the participants $\left(\mathrm{P}_{30}\right.$ $>90 \%)$. Recently, we showed the $\mathrm{P}_{30}$ for eGFRcreat (CKD-EPI) to be just $22 \%$ amongst Indian subjects. eG-
FRcys, which corrects for fallacies of creatinine-based equations, performed better at P30 of 75\%, but still fell short of the desired target [7]. The CKD-EPIcreat equation consistently overestimated the true GFR, which could explain the reclassification to a more adverse category in South Asians when eGFRcys was used, and also the inability of the creatinine-based equations to accurately predict outcomes. The reasons of the poor performance of creatinine-based equations could be related to the lower muscle mass, different body habitus and vegetarian dietary habits in South Asians.

The exact causal pathways linking CKD to CVD have not been established, but a plethora of mechanisms have been proposed. Given the strong evidence from several population groups, such relationship is likely to be present in South Asians as well. Perhaps the development of a more accurate ethnicity-specific equation, as has been done for Chinese, Japanese, Korean and Thai populations, will unmask the predictive value of eGFR for clinically important outcomes in South Asians. It is important to answer this question, so as to appropriately tailor the high-risk group approach for health care interventions and advocacy for funding for population segments. The ongoing Indian CKD Study [7] may help shed light on this.

Can such findings be explained simply on the basis of genetic differences? The human genome project educational material stated " 2 random individuals from any one group are almost as different (genetically) as any 2 random individuals from the entire world." Further, when individuals are sampled homogeneously from around the globe and not pre-selected based on particular regions or predefined attributes, the patterns of allele frequencies show a gradient across the world rather than the presence of discrete clusters [8]. Further studies are required to explore the interplay between genetic and nongenetic differences that have accumulated over millennia in different population groups and might be responsible for these biological differences. This understanding is important for developing future personalised medicine approaches.

\section{Disclosure Statement}

Dr. Vivekanand Jha reports grants from Department of Biotechnology, India, grants from Indian Council of Medical Research, Department of Bioechnology, India, Medical Research Council, UK, GSK, Baxter Healthcare, and personal fees from NephroPlus, Zydus Cadilla, and Biocon. Dr. Gopesh K. Modi has nothing to report. 


\section{References}

1 Naghavi M, Abajobir AA, Abbafati C, Abbas KM, Abd-Allah F, Abera SF, et al.; GBD 2016 Causes of Death Collaborators. Global, regional, and national age-sex specific mortality for 264 causes of death, 1980-2016: a systematic analysis for the Global Burden of Disease Study 2016. Lancet. 2017 Sep;390(10100): 1151-210.

2 Matsushita K, van der Velde M, Astor BC, Woodward M, Levey AS, de Jong PE, et al.; Chronic Kidney Disease Prognosis Consortium. Association of estimated glomerular filtration rate and albuminuria with all-cause and cardiovascular mortality in general population cohorts: a collaborative meta-analysis. Lancet. 2010 Jun;375(9731):2073-81.
3 Tillin T, Forouhi NG, McKeigue PM, Chaturvedi N.; SABRE Study Group. Southall And Brent REvisited: cohort profile of SABRE, a UK population-based comparison of cardiovascular disease and diabetes in people of European, Indian Asian and African Caribbean origins. Int J Epidemiol. 2012 Feb;41(1): 33-42.

4 Eastwood S, Chaturvedi N, Sattar N, Welsh P, Hughes AD, Tillin T. Impact of kidney function on cardiovascular risk and mortality: a comparison of South Asian and European cohorts. Am JNephrol.DOI: 10.1159/000503873.

5 Barbour SJ, Er L, Djurdjev O, Karim M, Levin A. Differences in progression of CKD and mortality amongst Caucasian, Oriental Asian and South Asian CKD patients. Nephrol Dial Transplant. 2010 Nov;25(11):3663-72.

6 de Zeeuw D, Parving HH, Henning RH. Microalbuminuria as an early marker for cardiovascular disease. J Am Soc Nephrol. 2006 Aug; 17(8):2100-5.

7 Kumar V, Yadav AK, Yasuda Y, Horio M, Kumar V, Sahni N, et al. Existing creatininebased equations overestimate glomerular filtration rate in Indians. BMC Nephrol. 2018 Feb;19(1):22.

8 Witherspoon DJ, Wooding S, Rogers AR, Marchani EE, Watkins WS, Batzer MA, et al. Genetic similarities within and between human populations. Genetics. 2007 May;176(1): 351-9. 